\title{
Distinct cell killing properties of the Drosophila reaper, head involution defective, and grim genes
}

\author{
John P. Wing ${ }^{1}$, Lei Zhou ${ }^{1,2}$, Lawrence M. Schwartz ${ }^{1}$ and \\ John R. Nambu ${ }^{1,3}$ \\ ${ }^{1}$ Biology Department, Morrill Science Center, University of Massachusetts at \\ Amherst, Amherst, Massachusetts 01003, USA \\ ${ }^{2}$ Current address: Howard Hughes Medical Institute, Departments of Biology \\ and Brain and Cognitive Sciences, Massachusetts Institute of Technology, \\ Cambridge, Massachusetts 02139, USA \\ ${ }^{3}$ corresponding author: tel: (413) 545 2782; fax: 4135453243 \\ e-mail: jnambu@bio.umass.edu
}

Received 23.3.98; revised 21.5.98; accepted 2.6.98

Edited by: B.A. Osborne

\begin{abstract}
The Drosophila reaper, head involution defective (hid), and grim genes play key roles in regulating the activation of programmed cell death. Two useful systems for studying the functions of these genes are the embryonic CNS midline and adult eye. In this study we use the Gal4/UAS targeted gene expression system to demonstrate that unlike reaper or hid, expression of grim alone is sufficient to induce ectopic CNS midline cell death. We also show that in both the midline and eye, grim-induced cell death is not blocked by the Drosophila anti-apoptosis protein Diap2, which does block both reaperand hid-induced cell death. grim can also function synergistically with reaper or hid to induce higher levels of midline cell death than observed for any of the genes individually. Finally we analyzed the function of a truncated Reaper-C protein which lacks the NH2-terminal 14 amino acids that are conserved between Reaper, Hid, and Grim. Ectopic expression of Reaper-C revealed cell killing activities distinct from full length Reaper, and indicated that the conserved NH2terminal domain acts in part to modulate Reaper activity.
\end{abstract}

Keywords: Drosophila; development; Gal4/UAS; Grim; Diap2

Abbreviations: hid, head involution defective; CNS, central nervous system; VUM, ventral unpaired median; IAPs, inhibitor-ofapoptosis proteins; RHG domain, reaper-hid-grim domain

\section{Introduction}

The Drosophila reaper, head involution defective (hid), and grim genes serve as key regulators of cell death activation (reviewed in McCall and Steller, 1997). These genes are all linked at the $75 \mathrm{C} 1,2$ region of the third chromosome and mutants that lack all three of these genes, such as $\mathrm{Df}(3 \mathrm{~L}) \mathrm{H} 99$, exhibit a blockade of essentially all cell death (White et al, 1994). hid-specific mutants as well as Df(3L)X25, which eliminates hid and grim but not reaper, also exhibit distinct disruptions of normal cell death patterns (Grether et al, 1995; Chen et al, 1996a; Zhou et al, 1997). The expression of reaper, hid, and grim is detected in, but not restricted to, doomed and dying cells, and ectopic expression of each gene can induce some cell types to die (White et al, 1994; Grether et al, 1995; Chen et al, 1996a; Nordstrom et al, 1996). These genes all function upstream of one or more caspases (Grether et al, 1995; Hay et al, 1995; Chen et al, 1996a; Pronk et al, 1996), as their activities are blocked by p35, a baculovirus caspase inhibitor (Bump et al, 1995; Xue and Horvitz, 1995). At present there is limited understanding of how these genes function to regulate the activation of cell death, as the precise biochemical activities of the Reaper, Hid, or Grim proteins are not yet known. Reaper shares some sequence similarity to the 'death domains' present in several vertebrate proteins involved in transducing cell death activation signals (Golstein et al, 1995), and Reaper appears to exhibit cytoplasmic localization in dying cells (Vucic et al, 1997a). Hid and Grim are both novel proteins whose subcellular localizations are not yet known. Significantly, the NH2terminal 14 amino acids of Reaper, Hid, and Grim all share sequence similarity, with Reaper and Grim being more closely related to each other than either is to Hid (Grether et al, 1995; Chen et al, 1996a). This similarity suggests that the $\mathrm{NH}-2$ terminal region may have common functions in all three proteins, and that elucidation of these functions is likely to be crucial for understanding the activities of these key cell death activators.

To begin to decipher the specific functions of reaper, hid, and grim, we initiated a series of in vivo functional analyses using the Drosophila embryonic central nervous system (CNS) midline as a model system (Zhou et al, 1995; 1997). The CNS midline consists of a well characterized set of $\sim 27$ cells that can be distinguished using molecular and morphological criteria (see Bossing and Technau 1993; Goodman and Doe, 1993). Among these cells are the midline glia, which are mostly located at the dorsal aspect of the ventral nerve cord, and the (ventral unpaired median) VUM neurons, which are situated ventrally. We and others have shown that the CNS midline exhibits a prominent, lineage-specific pattern of cell death during embryogenesis, as $2 / 3$ of the midline glia are normally eliminated, while few if any of the VUM neurons die (Sonnenfeld and Jacobs, 1995; Zhou et al, 1995). Genetic analyses of various $75 \mathrm{C} 1,2$ mutants that remove hid alone, hid and grim, or reaper, hid, and grim suggested that the combined functions of all three genes is necessary for the normal pattern of CNS midline cell death (Zhou et al, 1997). It was further demonstrated that ectopic expression of reaper or hid alone could not induce the death of midline cells that normally survive, however, co-expression of both genes resulted in a dramatic loss of midline cells (Zhou et al, 1997). These results provided the first indication that these genes can function synergistically, and suggest that they 
may act in a combinatorial fashion to regulate cell death in distinct cell types. An important question raised by these results is: what are the distinct cell death-inducing activities that these proteins may carry out?

In this study we use the Gal4/UAS targeted gene expression system (Brand and Perrimon, 1993) to show that grim can act in a distinct fashion from reaper or hid, as targeted expression of grim alone was sufficient to induce ectopic midline cell death. We also determined that grim cell killing activity is regulated differently than that of reaper or hid. Thus, the Drosophila inhibitor-of-apoptosis protein, Diap2, which blocks both reaper- and hid-induced cell death (Hay et al, 1995; Vucic et al, 1997a), fails to block grim-induced cell killing both in the midline and adult eye. grim can also act co-operatively with reaper or hid to induce higher levels of midline cell death than observed for expression of the individual genes.

We also analyzed the importance of the conserved NH2terminal region by generating a truncated Reaper-C protein which lacks amino acids $2-14$. The activity of this protein was assayed using the Gal4/UAS system both in the midline and the eye. In the midline, co-expression of Reaper-C with Hid or Grim induced very high levels of cell death, in excess with that observed for similar coexpression studies using wild-type Reaper. In the eye, expression of Reaper-C was able to induce moderate levels of cell death, however, the phenotype was less severe than observed with full length Reaper. Interestingly, unlike the effects of full length Reaper, Reaper-C induced eye cell death was only partially blocked by Diap2. Taken together these data suggest that the conserved $\mathrm{NH} 2$ terminal region may serve multiple roles in regulating the activity of Reaper protein in different cell lineages.

\section{Results \\ grim is a more potent inducer of CNS midline cell death than reaper or hid}

Previous studies indicated that ectopic expression of either reaper or hid alone is not sufficient to induce the death of midline cells that normally survive (Zhou et al, 1997). The more recently identified grim gene was not examined in that study, but is of interest because it shares some similarities to reaper and hid, but can induce cell death during earlier embryonic stages (Chen et al, 1996a). In order to analyze the ability of grim to induce midline cell death, we generated P[UAS-grim] transformant fly strains (see Materials and Methods). Two stains were analyzed, one with a viable second chromosome insertion, P[UAS-grim-2], and one with a viable third chromosome insertion, P[UAS-grim-3]. These strains were crossed to a $\mathrm{P}[52 \mathrm{~A}-$ gal4] strain that drives strong expression in the midline glia and VUM neurons from stage 11 onward (Zhou et al, 1997). This strain also carried a P[UASlacZ] chromosome to permit detection of CNS midline cells. The progeny embryos were analyzed via anti- $\beta$-gal immunocytochemistry and revealed clear cell killing phenotypes. For P[UAS-grim-2], the embryos typically exhibited a loss of all midline glia from 4-5 segments of the ventral nerve cord (Figure $1 \mathrm{~A}$ and $\mathrm{B}$ ) and also exhibited a fusion of several commissural axon bundles (Figure $1 \mathrm{C}$ and $\mathrm{D}$ ). This indicated that grim-induced killing of midline cells occurred in a rapid fashion during axonogenesis. Thus, unlike reaper or hid, expression of grim alone is sufficient to induce ectopic midline cell death. An even more severe cell death phenotype was observed with P[UAS-grim-3], where midline glia were absent in all but 1-2 segments and many of the VUM neurons were also eliminated (Figure 1E). Since previous studies indicated that grim cell killing effects are dosage sensitive (Chen et al, 1996a), the variability we observe likely reflects different levels of grim expression due to positional effects at the different P[UAS-grim] chromosomal insertion sites. For all further experiments we utilized the P[UAS-grim-2] strain. As expected, the grim-induced loss of midline cells was blocked by co-expression of the baculovirus protein p35 (Figure 1F), which inhibits caspase activity (Bump et al, 1995; Xue and Horvitz, 1995). Targeted expression of grim in a Df(3L)H99 background also resulted in ectopic midline cell death (data not shown), indicating that the functions of the endogenous reaper, hid, or grim genes are not necessary for grim-induced midline cell killing.

We then tested whether grim can act co-operatively with either reaper or hid. To address this issue we used P[UASreaper] and P[UAS-hid] strains (Zhou et al, 1997), along with P[UAS-grim-2], to examine the effects of co-expression of grim with reaper or hid. In embryos expressing both grim and reaper, nearly all the midline glia and VUM neurons were eliminated (Figure 1G). Similar effects on midline cell death were also detected with grim and hid co-expression (Figure $1 \mathrm{H}$ ). In addition, $\beta$-galactosidase-expressing cells were also eliminated in other embryonic tissues where $\mathrm{P}[52 \mathrm{~A}-\mathrm{ga} / 4]$ is expressed, such as the frontal sac as well as somatic and visceral musculature (data not shown). These phenotypes are distinct from the lack of ectopic cell death induced by reaper or hid alone (Zhou et al, 1997), or the moderate effect seen with P[UAS-grim-2]. Thus, grim can act co-operatively with both reaper and hid to induce high levels of midline cell death. Taken together with previous findings indicating co-operative interactions between reaper and hid (Zhou et al, 1997), and the overlapping yet distinct expression patterns of these genes (White et al, 1994; Grether et al, 1995; Chen et al, 1996a), these data strongly suggest that reaper, hid, and grim may all act in a combinatorial manner to induce death in cell lineagespecific manner.

\section{grim-induced cell death is not blocked by the anti- apoptotic protein Diap2}

The distinct cell killing abilities of grim raised the possibility that grim activity may be regulated differently than that of reaper or hid. Although grim-induced killing is blocked by the baculovirus p35 protein (Figure 1F; Chen et al, 1996a), we were interested in determining whether grim may be less sensitive to cellular apoptosis inhibitors. In Drosophila, there exist two related inhibitor-of-apoptosis proteins (IAPs), Diap1 and Diap2 (Hay et al, 1995), that belong to the IAP family of cell death repressor proteins expressed by viruses and animal cells (e.g. Crook et al, 1993; Birnbaum et al, 1994; Hay et al, 1995; Rothe et al, 1995; Liston et al, 1996). IAPs generally 
contain two or three conserved BIR domains located at the $\mathrm{NH}$-terminal and center portions of the protein, and most also contain a $\mathrm{COOH}$-terminal RING finger. Ectopic expression of Diap1 or Diap2 can block naturally occurring cell deaths in the developing adult eye, as well as suppress ectopic eye cell death induced by reaper or hid over-expression (Hay et al, 1995). Recent studies have further indicated that the BIR domain of Diaps can directly associate with Reaper protein, and that this interaction results in altered subcellular localization of Reaper (Vucic et al, 1997a).

To determine whether Diaps can also block grim-induced cell killing, we generated a P[UAS-diap2] strain (see Materials and Methods). In embryos where grim and diap2 were co-expressed in developing midline cells, there was a similar loss of midline glia from the nerve cord as detected for expression of grim alone (Figure 2A). This
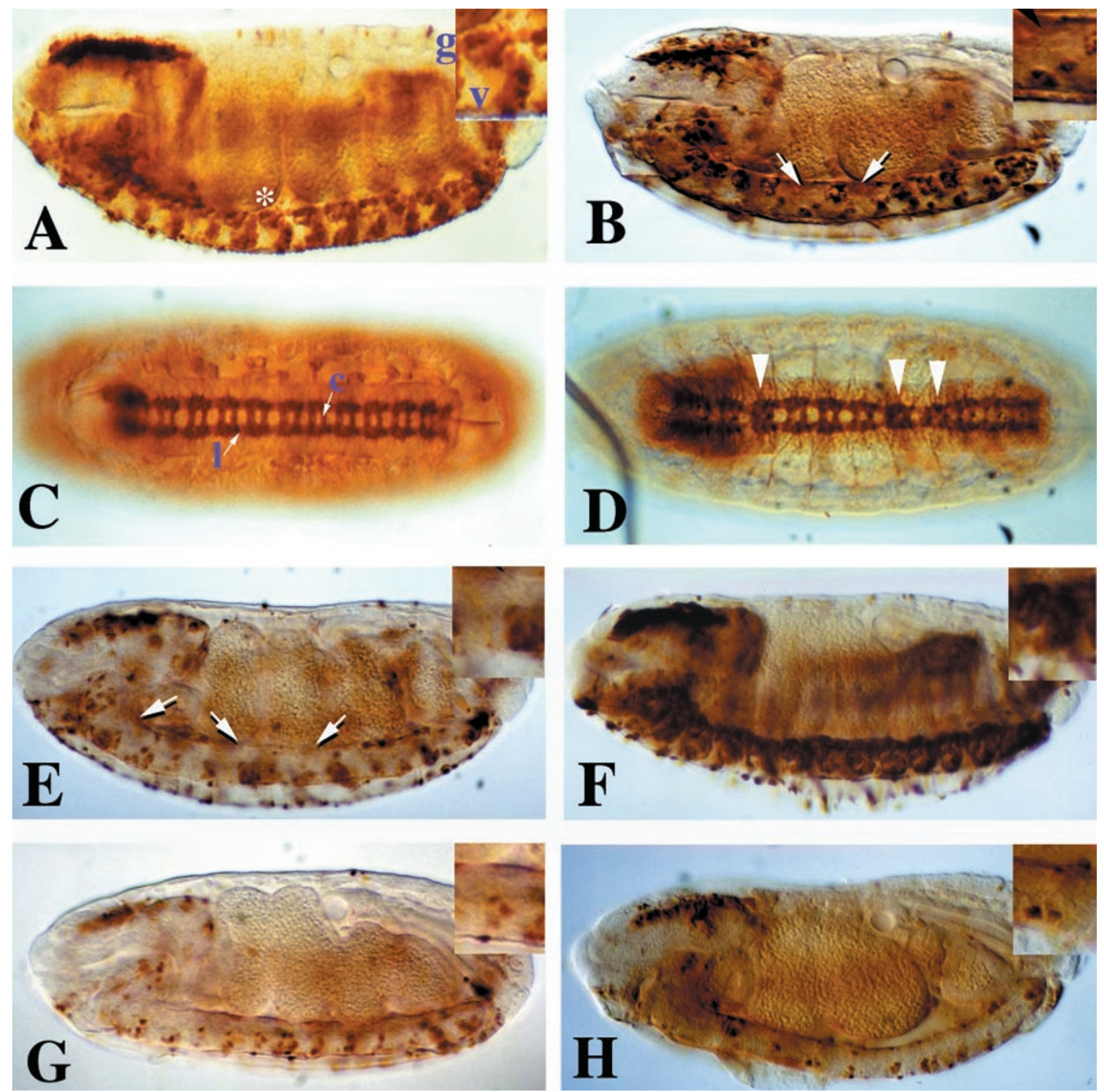

Figure 1 grim expression induces ectopic CNS midline cell death. Immunostaining of the following stage 16 embryos using either anti- $\beta$-galactosidase serum (A,B,E,F,G,H) or Mab BP102 (C,D): (A,C) P[UAS-lacZ]; P[52A-gal4]/+ (B,D) P[UAS-lacZ]; P[52A-gal4]/P[UAS-grim-2] (E) P[UAS-lacZ]; P[52A-gal4]/+; P[UASgrim-3]/+ (F) P[UAS-lacZ]; P[52A-gal4]/P[UAS-p35]; P[UAS-grim-3]/+ (G) P[UAS-reaper]/P[UAS-lacZ]; P[52A-gal4]/P[UAS-grim-2] and (H) P[UAS-lacZ]/P[UAShid]; P[52A-gal4]/P[UAS-grim-2]. (A) Note that $\beta$-gal expression is normally detected in both midline glia at the dorsal aspect of the nerve cord and the VUM neurons at the ventral region of the nerve cord. Inset shows a higher magnification view of the A2 segment (marked by *) and indicates midline glia ( $\mathrm{g}$ ) and VUM neurons (v). (C) The axon scaffold of these embryos exhibits a wild-type organization of longitudinal connectives (1) and commissural axon bundles (c). (B) Expression of P[UAS-grim-2] typically results in the loss of midline glia from 4-5 segments of the nerve cord (arrows), and (D) results in a fused commisure phenotype (arrowheads). (E) P[UAS-grim-3] induces a more pronounced loss of midline glia (arrows) as well as VUM neurons. (F) grim-induced ectopic midline cell death is blocked by co-expression of the baculovirus p35 protein. $(\mathbf{G}, \mathbf{H})$ Co-expression of grim and reaper $(\mathbf{G})$ or grim and hid $(\mathbf{H})$ result in a complete elimination of the midline glia as well as partial elimination of the VUM neurons. Compare this to the moderate midline cell death in (B), and absence of midline cell death induced by expression of reaper or hid alone (Zhou et al, 1997). All views are sagittal with anterior to left and dorsal up 
result indicated that diap2 is not capable of blocking griminduced midline cell killing. In contrast, diap2 was able to block the ectopic midline cell death induced by coexpression of reaper and hid, as midline glia were present in all segments (Figure 2B; see Zhou et al, 1997). The ability of diap2 to block cell killing induced by reaper and hid, but not grim, is a potentially important regulatory distinction between these three cell death activators.

We extended our analysis on the specificity of diap2 function by comparing its ability to inhibit grim-induced cell death in the developing adult eye. For these experiments we used a P[GMR-gal4] strain (see Materials and Methods) to drive Gal4 expression in differentiating photoreceptor cells in the eye imaginal disc (Hay et al, 1994). When driven by P[GMR-gal4], ectopic expression of reaper, hid, or grim alone, as well as reaper and hid together, resulted in lethality during late larval and pupal stages. This phenotype differs from previous studies where expression of reaper, hid, or grim were driven in the eye imaginal disc directly by GMR sequences, without the use of the Gal4/UAS system. Thus, P[GMR-reaper], P[GMR-hid], and P[GMR-grim] strains are all viable, but exhibit a dosage sensitive loss of eye cells (White et al, 1994, 1996; Grether et al, 1995; Hay et al, 1995; Chen et al, 1996a). Since the eye is not an essential organ, the lethality we observed using the Gal4/UAS system is likely due to expression of reaper, hid, or grim in cells outside of the eye imaginal disc. We examined whether diap2 could block the lethal effects of P[GMR-ga/4]-driven
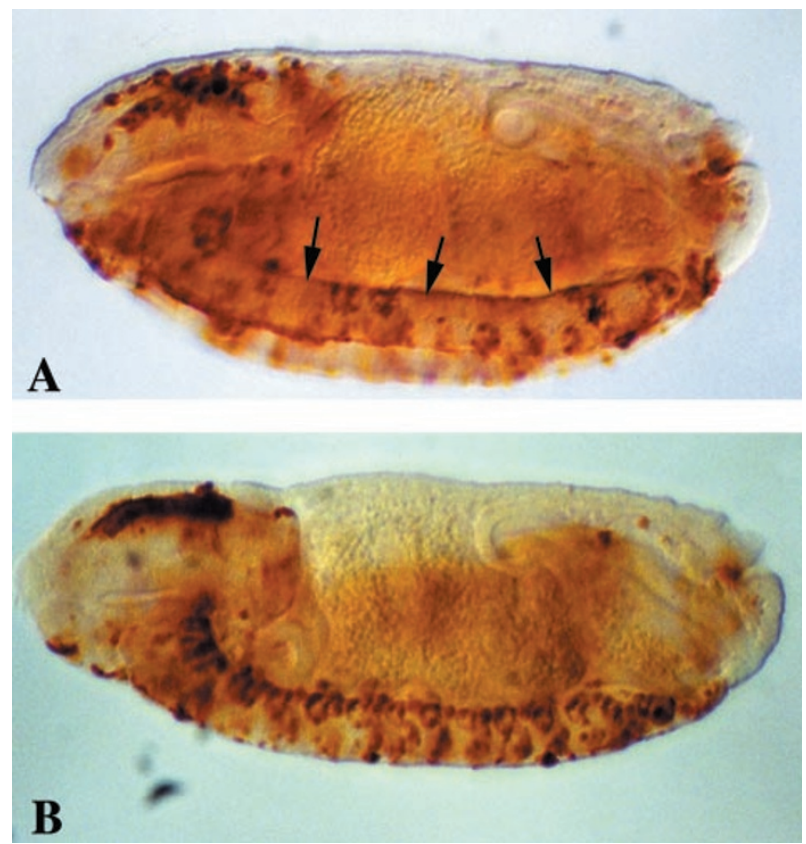

Figure 2 diap2 does not block grim-induced CNS midline cell death. Anti- $\beta$ galactosidase staining of the following stage 16 embryos: (A) P[UAS-lacZ]; $\mathrm{P}[52 \mathrm{~A}-$ gal4]/P[UAS-grim]; P[UAS-diap2]/+ and (B) P[UAS-lacZ]/P[UASreaper]-P[UAS-hid]; P[52A-gal4]/+; P[UAS-diap2]/+. Note that diap2 fails to block grim-induced midline cell death $(\mathbf{A})$, as several segments still lack midline glia (arrows). diap2 does block midline cell deaths induced by coexpression of reaper and hid, (B), as wild-type numbers of cells are detected. All views are sagittal with anterior to left and dorsal up expression of these genes. Co-expression of diap2 was essentially unable to rescue the lethality of ectopic grim expression, as less than $1 \%$ of pupae eclosed; the few adults that did hatch exhibited a complete elimination of eye tissue (Compare Figure $3 A$ and $D$ to $B$ and $E$ ). This effect is likely dosage-sensitive, as high levels of diap2 expression using the Gal4/UAS system could block the weak eye cell death phenotype of a single copy of a P[GMR-grim] chromosome (see Materials and Methods). In contrast, using the Gal4/UAS system diap2 was able to fully rescue the lethality induced by ectopic expression of reaper or hid alone (data not shown), as well as co-expression of reaper and hid together (Figure $3 \mathrm{C}$ and $\mathrm{F}$ ). In addition, $97 \%$ of these flies exhibited normal eye morphology while only $3 \%$ exhibited a roughened eye phenotype. These results confirm functional specificity for diap2, and indicate that grim exhibits distinct cell killing properties than reaper or hid both in the CNS midline and adult eye.

\section{Functions of the conserved RHG domain}

The Reaper, Hid, and Grim proteins all share a conserved 14 amino acid region at their NH2-termini (Grether et al, 1995; Chen et al, 1996a), which we propose to refer to as the RHG domain, for Reaper, $\underline{H}$ id, and Grim. In particular, the RHG domains of Reaper and Grim are $71 \%$ identical, while both are less conserved with that of Hid. This sequence similarity suggests the RHG domain carries out common functions in these three proteins. Previous studies on truncated Reaper proteins in insect tissue culture cells suggested that the RHG domain might be important for normal levels of Reaper activity (Chen et al, 1996b; Vucic et al, 1997b). Because different cell types, such as the embryonic CNS midline and adult eye, exhibit distinct sensitivity to ectopic Reaper expression, we wished to further address this issue in developing fly tissues. Consequently we generated P[UAS-reaper-C] fly strains that express a truncated version of Reaper protein lacking residues $2-14$, and compared the cell killing abilities of this Reaper-C protein to wild-type Reaper. Two P[UAS-reaper-C] strains were obtained, one with a viable insertion on the $X$ chromosome, P[UAS-reaper-C-X], and one with a viable insertion on the third chromosome, P[UAS-reaper-C-3]. Both strains exhibited similar activities.

As with wild-type Reaper, targeted midline expression of Reaper-C did not result in any ectopic cell death (Figure 4A). In addition, we did not detect any excess midline glia, indicating that Reaper- $\mathrm{C}$ did not act as a dominant negative to block normal midline cell deaths. We then tested whether Reaper-C could function co-operatively with either Hid or Grim. Co-expression of Reaper-C and Grim resulted in a loss of both midline glia and VUM neurons from all segments of the nerve cord (Figure 4B), a level of cell killing much greater than that observed for expression of either Reaper-C or Grim alone. Interestingly, this level of cell death was also more severe than that observed for coexpression of wild-type Reaper and Grim (Figure 1G), suggesting that Reaper-C may possess enhanced midline cell killing activity. Co-expression of Reaper-C with Hid also resulted in a synergistic elimination of midline glia and VUM neurons from most segments (Figure 4C), an effect which 

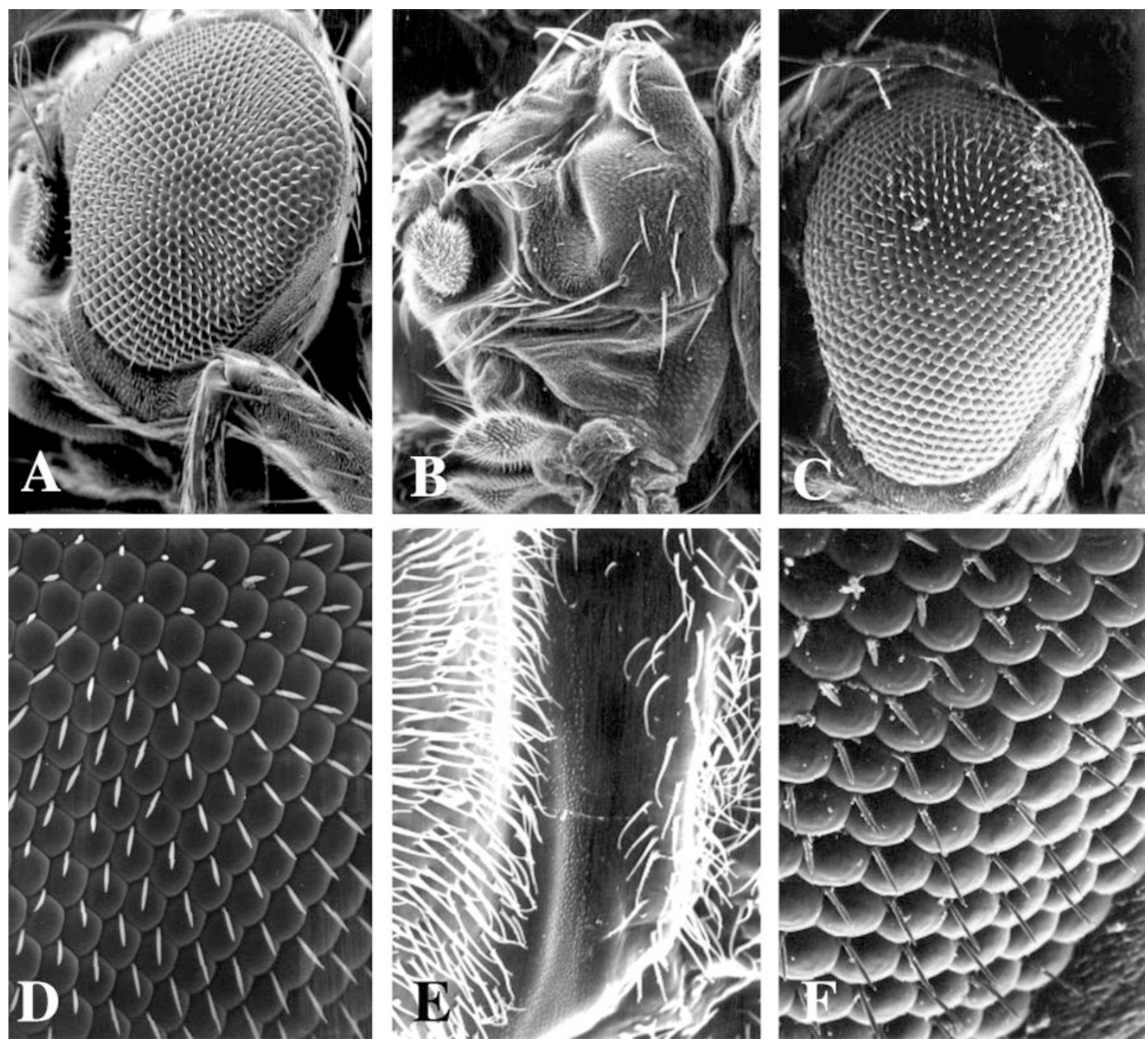

Figure 3 diap2 specifically fails to block grim-induced eye cell death. (A,D) Wild-type adult eye. Note ordered array of ommatidia and bristles. (B,E) diap2 failed to rescue the lethality of P[GMR-ga/4]/P[UAS-grim-2] animals, as less than $1 \%$ of the P[GMR-gal4]/P[UAS-grim2];P[UAS-diap2]/+ pupae eclosed and these adults exhibited an absence of ommatidia. (C,F) diap2 did rescue the lethality of P[UAS-reaper]-P[UAS-hid]; P[GMR-gal4]/+ animals: 97\% of the P[UAS-reaper]-P[UAShid]; P[GMR-gal4]/+; P[UAS-diap2]/+ adults exhibited a normal eye phenotype and $3 \%$ exhibited a roughened eye. All panels are S.E.M. micrographs at magnifications of: $(A, B, C) \times 200,(D) \times 700,(E) \times 2000,(F) \times 1000$

differed from the lack of ectopic midline cell death induced by expression of either Reaper-C (Figure $4 \mathrm{~A}$ ) or Hid alone (see Zhou et al, 1997). The extent of cell death induced by co-expression of Reaper-C with Hid was also more severe than that observed for co-expression of wild-type Reaper and Hid (see Zhou et al, 1997). Taken together, these data indicate that the RHG domain of Reaper is not required for co-operative interactions with Hid or Grim, and suggest that one function of the RHG domain may be to modulate Reaper killing activity.

We also analyzed the cell killing ability of Reaper-C in the adult eye using the P[GMR-ga/4] strain. Unlike the lethal phenotype observed for wild-type Reaper, flies ectopically expressing Reaper-C were viable, and exhibited a dosage-sensitive elimination of eye tissue. Flies heterozygous for P[GMR-ga/4] and homozygous for P[UASreaper-C-X] exhibited a significant reduction of eye tissue, with a loss of ommatidia and disorganization of mechanosensory bristles (Figure 5A, B). In contrast, females heterozygous for P[UAS-reaper-C-X] exhibited normal eye morphology (data not shown). Thus, Reaper-C is capable of inducing eye cell death, although the absence of the RHG domain results in less cell killing activity than wildtype Reaper. This result contrasts with the stronger synergistic killing of CNS midline cells by Reaper-C than wild-type Reaper, and implies that differences in the levels of Reaper and Reaper-C proteins are unlikely to be responsible for these contrasting cell death phenotypes. The different effects of Reaper-C in the midline and eye also suggest that there may exist tissue-specific differences in the functions of the RHG domain.

We then examined whether Diap2 was capable of blocking Reaper-C-induced eye cell death. Co-expression of Reaper-C and Diap2 via P[GMR-gal4] resulted in only a 
partial repression of Reaper-C activity, as $50 \%$ of male flies exhibited a wild-type eye while $50 \%$ exhibited a roughened eye phenotype that was similar to, but less severe than that of Reaper-C alone (Figure 5C, D). This finding indicates that Diap2 is less effective in blocking the activity of Reaper-C than full length Reaper, suggesting that the cell death inhibitory activities of Diap2 may be mediated, at least in part, through $\mathrm{RHG}$ domain.

\section{Discussion}

\section{Distinct cell killing properties of grim-reaper genes}

The reaper, hid, and grim genes encode important cell death activators whose functions are just beginning to be
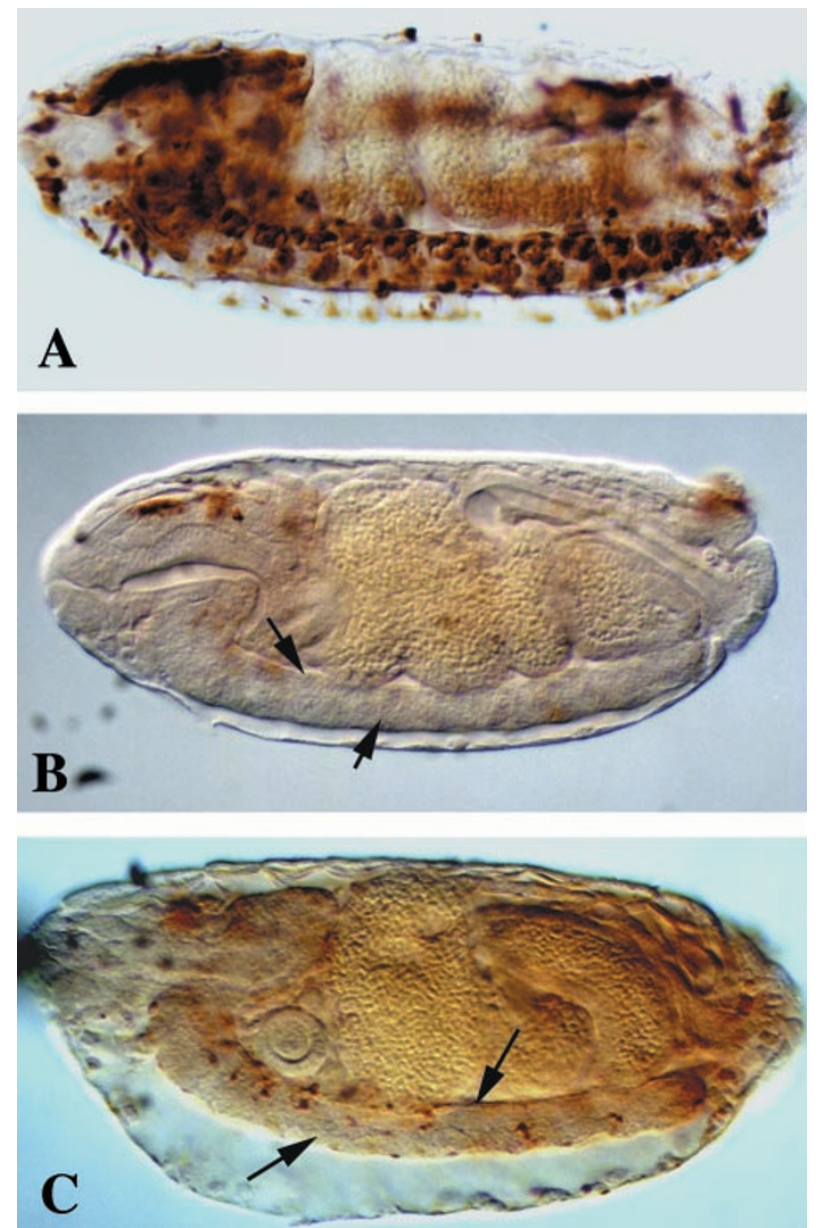

Figure 4 A Reaper-C protein lacking the conserved RHG domain can act cooperatively with Hid and Grim to induce CNS midline cell death. (A-C) Anti- $\beta$ galactosidase staining of the following stage 16 embryos: (A)P[UAS-lacZ]; $\mathrm{P}[52 \mathrm{~A}-$ gal4]/+; P[UAS-reaper-C-3]/+ (B) P[UAS-lacZ]; P[52A-gal4]/P[UASgrim-2]; P[UAS-reaper-C-3]/+ and (C) P[UAS-lacZ/P[UAS-hid]/; P[52A-gal4]/+; P[UAS-reaper-C-3]/+. Note that Reaper-C expression alone does not result in any ectopic midline cell death (A). Co-expression of Reaper-C with Grim (B) or Hid (C) results in severe loss of both midline glia and VUM neurons (arrows). This phenotype is more severe than that seen when full length Reaper is expressed with Grim (compare to Figure 1G) or Hid (see Zhou et al, 1997). All views are sagittal with anterior to left and dorsal up elucidated. Because these genes all function to regulate cell death, share conserved RHG domains, and are linked at $75 \mathrm{C} 1,2$, we propose to refer to them as genes of the grimreaper locus. In this study, we show that in contrast to reaper and hid, ectopic expression of grim alone is sufficient to induce the death of CNS midline cells that normally survive. This finding indicates that Grim protein has cell killing capabilities distinct from those of Reaper and Hid. Perhaps Grim is able to access different components of the cell death machinery than Reaper or Hid, or access the same components in a distinct fashion. Another possibility is that Grim's activity may be regulated in a distinct fashion from Reaper or Hid. Consistent with this hypothesis we find that Grim is unique in that cell killing effects are not effectively inhibited by the cellular anti-apoptotic protein Diap2. Thus, while Diap2 can block both Reaper- and Hid-induced cell killing (Hay et al, 1995; Vucic et al, 1997a; this study), it does not block Grim-induced cell death. Although the mechanisms through which the Diaps block Reaper- and Hid-induced cell deaths are not yet clear, the BIR domains of laps can directly associate with Reaper, suggesting potential direct inhibitory interactions (Vucic et al, 1997a). Our results suggest this interaction likely requires the conserved $\mathrm{RHG}$ domain, as cell killing by a truncated Reaper-C protein was only partially blocked by Diap2. Our results further imply that residues shared between the RHG domains of Reaper and Hid, but which differ from Grim, may be crucial for Diap2 specificity. In this regard there are three such positions, at residues \#3, 5, and 6 , that are Valine, Phenylalanine, and Tyrosine in Reaper and Hid, and Isoleucine, Tyrosine, and Phenylalanine in Grim (Chen et al, 1996a). Previous studies indicated that mutation of the Phenylalanine or Tyrosine residues to Alanine reduced Reaper cell killing activity in insect tissue culture cells (Vucic et al, 1997b), and it will also be of interest to examine the effect of mutating these residues on Reaper or Grim killing activity and regulation by Diap2 in developing fly tissues.

Studies of reaper, hid, and grim functions using the embryonic midline as a model system have revealed cooperative interactions between all three of these genes. An important goal will be to define the mechanisms underlying these interactions. One model is that reaper and hid act together to activate a diap2-repressible cell death pathway. In contrast, grim can act co-operatively with reaper or hid but also can function in a distinct diap2-independent pathway. This differs somewhat from the actions of grimreaper genes in the adult eye, where each gene is individually capable of inducing cell death and no synergistic actions have been noted (White et al, 1994; Grether et al, 1995; Hay et al, 1995; Chen et al, 1996a). This difference suggests there may exist tissue-specific pathways through which these genes activate the cell death machinery. These pathways all appear to ultimately converge on one or more caspases, as cell killing by all three genes, alone or in combination, is blocked by p35 (Grether et al, 1995; Hay et al, 1995; Chen et al, 1996a; Vucic et al, 1997; Zhou et al, 1997). The ability of grimreaper genes to functionally interact suggests that they may all function in a combinatorial fashion in distinct populations of dying cells. Interestingly, while vertebrate 
homologs of grim-reaper genes have not yet been identified, like other cell death activators, reaper-induced cell killing can result in increased ceramide production (Pronk et al, 1996), and can cause release of cytochrome
C to induce apoptosis in Xenopus egg cell free extracts (Evans et al, 1997). These results suggest that grimreaper genes may act through conserved signal transduction pathways.
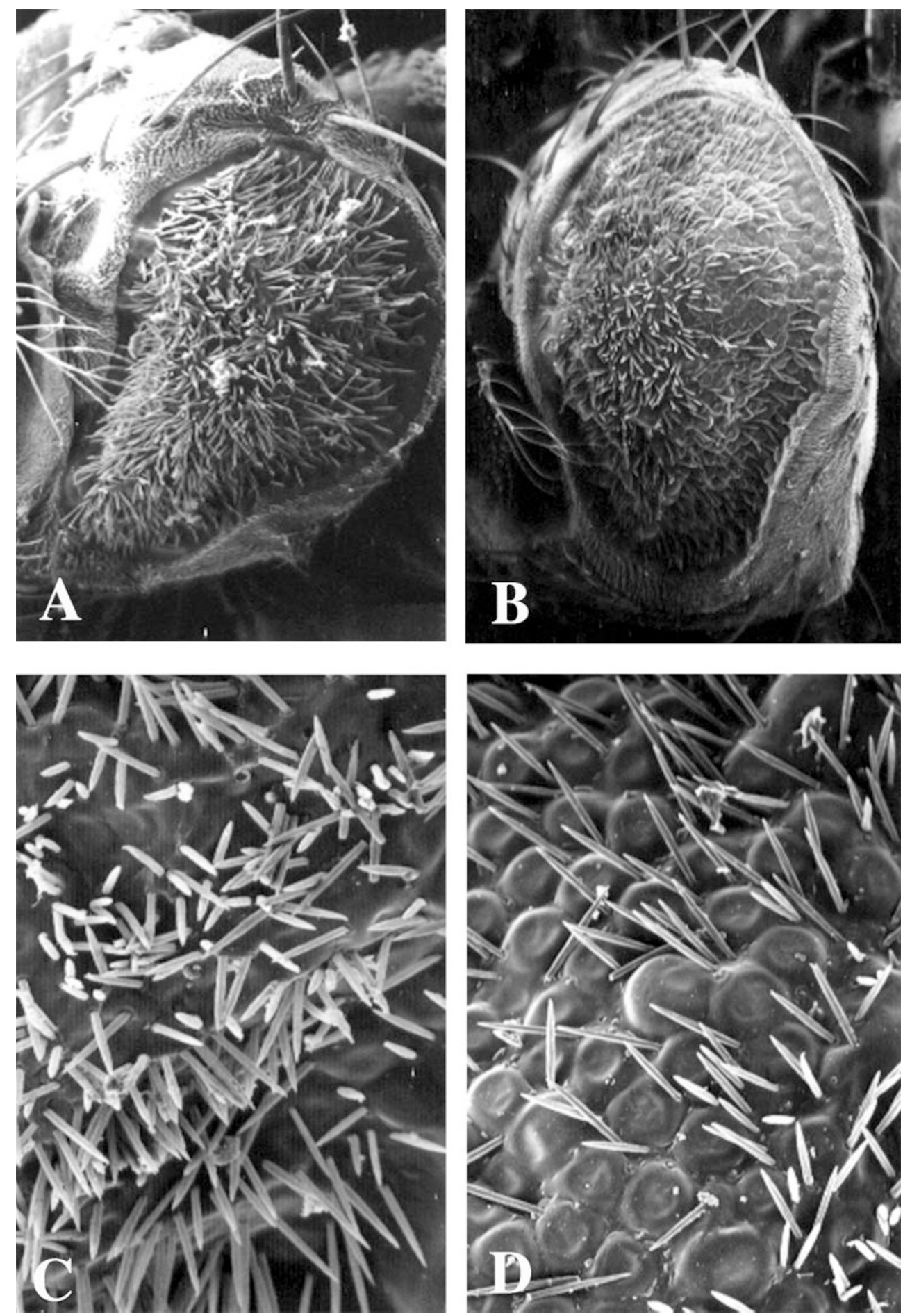

Figure 5 Reaper-C can induce cell death in the adult eye which is not abolished by Diap2. (A,C) A male P[UAS-reaper-C-X]; P[GMR-gal4]/+ fly exhibiting a decrease in eye size (compare to Figure 3A). Note also a loss of ommatidia and disorganization of bristles. (B,D) Approximately 50\% of the male P[UAS-reaper-C$\mathrm{X}$; P[GMR-ga/4]/+; P[UAS-diap2]/+ flies also exhibited a reduced eye phenotype that was less severe than P[UAS-reaper-C-X]; P[GMR-ga/4]/+ flies 


\section{Multiple functions of RHG domain}

While the grim-reaper genes are crucial for inducing cell death, the biochemical activities of the corresponding proteins are as yet unknown. Reaper does exhibit limited homology to the 'death domain', a protein/protein interaction motif present in a number of signaling proteins that function in cell death pathways (Golstein et al, 1995). However, a number of recent studies suggest that Reaper may function differently than the death domains of the vertebrate Fas, FADD, TNFR1 proteins (Chen, 1996b; Kondo et al, 1997; Vucic et al, 1997b), and mutation of several key residues essential for the functions of vertebrate death domain proteins did not eliminate Reaper activity (Chen et al, 1996b; Vucic et al, 1997b). One potential clue to the functions of Reaper, Hid and Grim is that they all share a conserved $\mathrm{RHG}$ domain at their NH2-termini. By analyzing the activity of a truncated Reaper-C polypeptide lacking this conserved region, we determined that the RHG domain is not required for the co-operative killing of CNS midline cells with Hid or Grim, or the killing of adult eye cells. Thus the $\mathrm{COOH}$-terminal 50 amino acids possess clear cellkilling activity. Although the functions of the Reaper RHG domain are not yet known, it could be important for Reaper stability, as a similarly truncated Reaper polypeptide exhibited low levels of accumulation in tissue culture cells (Vucic et al, 1997a). However, this explanation is not sufficient to describe the effects of co-expression of Reaper-C with Hid or Grim in the midline, which led to higher levels of cell death than observed for similar co-expression studies using full length Reaper. Our data support a hypothesis where the RHG domain has multiple functions in regulating Reaper activity. In the CNS midline, the Reaper RHG domain functions largely to restrict Reaper activity, perhaps by association with Diaps. In the adult eye, the RHG domain is required both for full cell killing activity and repression by Diap2. One question that will be of interest to address is whether the respective $\mathrm{RHG}$ domains of Reaper, Hid, and Grim confer specific cell killing and regulatory properties.

\section{Developmentally specific killing by grim-reaper genes}

Ectopic expression of grim-reaper genes have been used to induce cell death in several developmental contexts. Our studies on the CNS midline have indicated that cell death is most efficiently induced by co-expression of these genes. In addition to the midline, we have also observed that other embryonic cell types are relatively insensitive to the individual expression of Reaper, Hid, and to a lesser extent, Grim (JPW, LZ, LMS, JRN unpublished observations). These findings are in contrast to the effects of ectopic expression of these genes during postembryonic stages. For example, expression of any one of these genes is sufficient to induce adult eye cell death (White et al, 1994; Hay et al, 1995; Grether et al, 1995; Chen et al, 1996a), and ectopic expression of reaper or hid alone can also effectively induce the death of distinct sets of neurosecretory cells during metamorphosis (McNabb et al, 1997; Robinow et al, 1997). In addition, there are no apparent synergistic effects of co-expression of these genes in post- embryonic stages, and genetic enhancer/suppressor screens for mutations that influence the eye killing phenotype of reaper or hid have not revealed interactions between grim-reaper genes (Hay et al, 1995). These differences suggest there may exist distinct cell killing mechanisms utilized during different developmental stages. Indeed, White et al. (1996) have shown that 12-18 h old embryos and late stage pupae exhibit a reduced sensitivity to ectopic reaper expression.

It is possible that the differences observed between ectopic cell killing by grim-reaper genes in embryonic and post-embryonic stages is that there is simply more time for the corresponding proteins to accumulate and exert their cell killing functions during later stages. For example, genes under control of the cloned GMR sequences are expressed for over $50 \mathrm{~h}$ during eye imaginal disc differentiation in third instar larvae (Moses and Rubin, 1991; Hay et al, 1994), longer than the entire duration of embryogenesis. However, in our embryonic CNS midline killing experiments the cells are likely exposed to high levels of ectopic reaper, hid, or grim expression for at least $10 \mathrm{~h}$. Both in vivo and in vitro studies have indicated that for many dying cells, cell death initiation can be detected within 1-2 h after reaper expression (White et al, 1994; Nordstrom et al, 1996; Vucic et al, 1997b). These data strongly suggest that timing alone is not sufficient to account for inefficient embryonic cell killing. Instead, they suggest that there may be important differences in the available components of the cell death machinery during different developmental stages. Similar differences may also exist between different cell types, as lineage-specific differences in the sensitivity to expression of grim-reaper genes have also been noted, e.g. between the midline glia and VUM neurons. In this regard, as occurs in vertebrates, where expression of multiple caspases and $\mathrm{Bcl}-2$ family members provide lineage-specific regulation of apoptotis (see White, 1996), the three linked and related genes of the grim-reaper locus may provide enhanced flexibility in regulating the activation of cell death in distinct developmental and physiological contexts.

In Drosophila, embryogenesis is quite rapid and accompanied by a complex series of morphogenic movements, such as germ band extension and retraction, and head involution, that require tight regulation of cell division, differentiation, migration, and death. The pace of embryogenesis necessitates the efficient elimination of unwanted cells, requiring fast-acting mechanisms to mediate cell death. However, the generation of many embryonic stem cells that give rise to large numbers of progeny also requires mechanisms that ensure a very strict control over cell death activation. Thus, aberrant deletion of small numbers of cells in the embryo could have more pronounced effects than a similar cell loss during later developmental stages. This may have led to the establishment of stage- and lineage-specific regulatory mechanisms used to modulate the activity of grim-reaper gene products. It is likely that elucidating the mechanisms underlying these differences in cell death activation will ultimately have important implications for understanding and manipulating cell death in a variety of organisms. 


\section{Materials and Methods}

\section{Fly strains and genetic crosses}

P[UAS-grim] strains were generated by cloning a full length grim cDNA (provided by J Abrams) into the EcoRI site of the pUAST vector (Brand and Perrimon, 1993). P element mediated germline transformation into $w^{1118}$ host embryos was performed as originally described by Spradling and Rubin (1982). Two transformant strains were obtained, one with a viable insertion on the second chromosome and the other with a lethal insertion on the third chromosome. A viable third chromosome insertion was subsequently obtained via mobilization of the second chromosome $P$ element insertion using $\Delta 2-3$ as a source of transposase (Robertson et al, 1988). A P[UAS-diap2] strain was generated using a similar approach. A full length diap2 cDNA clone (LD04614) was obtained from the Berkeley Drosophila Genome Project and digested with $E c o R I$ and Xhol. This fragment was then cloned into the pUAST vector and microinjected into $w^{1118}$ host embryos. A viable insertion on the third chromosome was obtained and used for all studies described here.

The P[UAS-reaper-C] construct was generated by amplifying a DNA fragment encoding amino acids $15-65$ of the Reaper protein from a full length reaper cDNA clone in the pBluescript vector (Stratagene) using a reaper-specific primer: 5'-GCCCGAATTCATTAAATTAAATACCGCCATGCGGGAGGCGGAGCAG-3', and the T3 primer for pBluescript. This fragment was digested with $E c o R I$ and cloned into the pUAST vector. P[UAS-reaper-C] transformant strains were then generated as described above. Two viable insertions were obtained, one on the $\mathrm{X}$ chromosome and one on the third chromosome.

The P[52A-gal4] strain (Zhou et al, 1997) drives strong CNS midline expression from stage 11 onward (stages defined in CamposOrtega and Hartenstein, 1997). Expression is also present in several other embryonic tissues, including the frontal sac as well as visceral and somatic muscles. A P[UAS-lacZ]; P[52A-ga/4]/CyO strain was generated using an $X$ chromosome linked $P$ [UAS-lacZ] insertion provided by A Brand. Several P[UAS- $p 53$ ] strains were kindly provided by $B$ Hay. For these experiments we utilized a viable 2 nd chromosome insertion. The P[GMR-grim] strain was provided by Hermann Steller. It contains a viable second chromosome insertion that yields a mild loss of ommatidia as a heterozygote. The P[GMR-gal4] strain (a.k.a. $w^{*}$; $\mathrm{P}\{\mathrm{W}+\mathrm{mC}=\mathrm{GAL} 4-$ ninaE.GMR $\} 12)$ drives strong expression in and behind and morphogenic furrow in the developing eye imaginal disc; it was generated by M Freeman and obtained from $\mathrm{K}$ Matthews at the Indiana Stock Center. X-linked P[UAS-reaper], P[UAS-hid], and P[UAS-reaper]-P[UAS-hid] strains were previously described in Zhou et al, (1997). All grim-reaper gene combinatorial experiments were performed using the viable second chromosome insertion strain, P[UAS-grim-2].

\section{Immunocytochemistry and in situ hybridization}

For immunocytochemical analyses, embryos were collected and fixed using the PEMS/formaldehyde procedure of Patel (1994). A mouse monoclonal anti- $\beta$-galactosidase antibody (Promega) was used at $1: 1000$ to detect lacZ expression, and labeling was identified using a biotinylated horse anti-mouse antibody (Vector Labs) and strepavidinHRP. The monoclonal antibody BP102 was obtained from the Developmental Studies Hybridoma Bank and used at $1: 5$ to visualize CNS axon pathways. Labeling was detected using a biotinylated horse anti-mouse (Vector Labs) and strepavidin-HRP. Stained embryos were dehydrated in an ethanol series, cleared in methyl salicylate, and mounted in Permount (Fisher).

\section{Electron microscopy}

For EM analyses, adult Drosophila tissues were prepared by Dr. Lucy Yin at the University of Massachusetts Central Microscopy Facility. Briefly, tissue samples were fixed with $2 \%$ glutaraldehyde in $0.1 \mathrm{M}$ sodium cacodylate buffer $\mathrm{pH} 7.2$ for $3 \mathrm{~h}$ at room temperature. After dehydration through a graded ethanol series, they were critical point dried in a Polaron CPD unit. Samples were sputter coated with gold/ palladium and examined and photographed using a JEOL 5400 scanning electron microscope.

\section{Acknowledgements}

We thank John Abrams for providing the grim cDNA clone, Aletta Schnitzler for assistance in generating the P[UAS-reaper-C] strain, and the Berkeley Drosophila Genome Project for providing the diap2 cDNA clone. We are also grateful to Lucy Yin, for electron microscopy work, Hermann Steller, Andrea Brand, Bruce Hay, and Kathy Matthews, for providing fly stocks, and Ashim Mukherjee, for comments on the manuscript. This work was supported by NIH grant NS32251 to JRN and $\mathrm{NIH}$ grant AG55118 to LMS and JRN.

\section{References}

Birnbaum MJ, Clem RJ and Miller LK (1994) An apoptosis-inhibiting gene from a nuclear polyhedrosis virus encoding a peptide with Cys/His sequence motifs. J. Virol. 68: 2521-2328

Bossing T and Technau GM (1993) The fate of the CNS midline progenitors in Drosophila as revealed by a new method for single cell labeling. Development 120: $1895-1906$

Brand AH and Perrimon N (1993) Targeted gene expression as a means of altering cell fates and generating dominant phenotypes. Development 118: 401-415

Bump NJ, Hackett M, Hugunin M, Seshagiri S, Brady K, Chen P, Ferenz C, Franklin S, Ghayur T, Li P, Licari P, Mankovich J, Shi L, Greenberg AH, Miller LK and Wong WW (1995) Inhibition of ICE family proteases by baculovirus antiapoptotic protein p53. Science 269: 1885-1888

Campos-Ortega JA and Hartenstein V (1997) in The Embryonic Development of Drosophila melanogaster, Springer-Verlag

Chen P, Nordstrom W, Gish B and Abrams JM (1996a) grim, a novel cell death gene in Drosophila, Genes \& Dev. 1014: 1773-1782

Chen P, Lee P, Otto L and Abrams J (1996b) Apoptotic activity of REAPER is distinct from signaling by the tumor necrosis factor receptor 1 death domain. J. Biol. Chem. 27142: 25735-25737

Crook NE, Clem RJ and Miller LK (1993) An apoptosis-inhibiting baculovirus gene with a zinc finger-like motif. J. Virol. 67: 2168-2174

Evans EK, Kuwana T, Strum SL, Smith JJ, Newmeyer DD and Kornbluth S (1997) Reaper-induced apoptosis in a vertebrate system. EMBO J. 16: 7372-7381

Golstein P, Marguet D and Depraetere V (1995) Homology between Reaper and the cell death domains of Fas and TNFR1. Cell 81: 185

Goodman CS and Doe CQ (1993) Embryonic development of the Drosophila central nervous system. In The Development of Drosophila melanogaster, Cold Spring Harbor Press: 1131 - 1206

Grether ME, Abrams JM, Agapite J, White Kand Steller H(1995) The head involution defective gene of Drosophila melanogasterfunctions in programmed cell death. Genes \& Dev. 9: $1694-1708$

Hay BA, Wolff T and Rubin GM (1994) Expression of baculovirus P35 prevents cell death in Drosophila. Development 120: 2121-2129

Hay BA, Wassarman DA and Rubin GM (1995) Drosophila homologs of baculovirus inhibitor of apoptosis proteins function to block cell death. Cell 83: 1253-1262

Kondo T, Yokokura T and Nagata S (1997) Activation of distinct caspase-like protease by Fas and Reaper in Drosophila cells. Proc. Natl. Acad. Sci. USA 94: $11951-11956$

Liston P, Roy N, Tamai K, Lefebre C, Baird S, Cherto-Horvat G, Farahani R, McLean M, Ikedal J, MacKenzie A and Korneluk RG (1996) Suppression of apoptosis in mammalian cells by NAIP and a related family of IAP genes. Nature 379: $349-$ 353 
McCall K and Steller H (1997) Facing death in the fly: Genetic analysis of apoptosis in Drosophila. Trends Genet. 13: 222-226

McNabb S, Baker JD, Agapite J, Steller H, Riddiford LM and Truman JW (1997) Disruption of a behavioural sequence by targeted death of peptidergic neurons in Drosophila. Neuron 19: 813-823

Moses K and Rubin GM (1991) glass encodes a site-specific DNA-binding protein that is regulated in response to positional signals in the developing Drosophila eye. Genes \& Dev. 5: 583-593

Nordstrom W, Chen P, Steller H and Abrams JM (1996) Activation of the reaper gene during ectopic cell killing in Drosophila. Dev. Biol. 180: 213-226

Patel NH (1994) Imaging neuronal subsets and other cell types in whole-mount Drosophila embryos and larvae using antibody probes. In LSB Goldstein, EA Fyrberg (eds). Drosophila melanogaster: Practical uses in cell and molecular biology. Academic Press 446-485

Pronk GJ, Ramer K, Amiri P and Williams LT (1996) Requirement of an ICE-like protease for induction of apoptosis and ceramide generation by REAPER. Science 271: $808-810$

Robertson HM, Preston CR, Phillis RW, Johnson-Schlitz DM, Benz WK and Engels WR (1988) A stable genomic source of P-element transposase in Drosophila melanogaster. Genetics 118: 461-471

Robinow S, Draizen TA and Truman JW (1997) Genes that induce apoptosis: Transcriptional regulation in identified, doomed neurons of the Drosophila CNS. Dev. Biol. 190: 206-213

Rothe M, Pan M-G, HenzelWJ, Ayres TM and Goeddel DV (1995) The TNFR2-TRAF signaling complex contains two novel proteins related to baculoviral inhibitor of apoptosis proteins. Cell 83: 1243-1252
Sonnenfeld MJ and Jacobs JR (1995) Apoptosis of the midline glia during Drosophila embryogenesis: a correlation with axon contact. Development 121: 569-578

Spradling AC and Rubin GM (1982) Transposition of cloned P elements into Drosophila germ line chromosomes. Science 218: $341-347$

Vucic D, Kaiser WJ, Harvey AJ and Miller LK (1997a) Inhibition of Reaper-induced apoptosis by interaction with inhibitor of apoptosis proteins (IAPs). Proc. Natl. Acad. Sci. USA 94: 10183-10188

Vucic D, Seshagiri S and Miller LK (1997b) Characterization of Reaper- and FADDinduced apoptosis in a lepitopteran cell line. Mol. Cell. Biol. 17: 667-676

White K, Grether ME, Abrams JM, Young L, Farrell K and Steller H (1994) Genetic control of programmed cell death in Drosophila. Science 264: 677-683

White $E$ (1996) Life, death, and the pursuit of apoptosis. Genes \& Dev. 10: 1-15

White K, Tahaoglu E and Steller H (1996) Cell killing by the Drosophila gene reaper. Science 271: $805-807$

Xue D and Horvitz HR (1995) Inhibition of the Caenorhabditis elegans cell-death protease CED-3 by a CED-3 cleavage site in baculovirus p35 protein. Nature 377: $248-251$

Zhou L, Hashimi H, Schwartz LM and Nambu JR (1995) Programmed cell death in the Drosophila central nervous system midline. Curr. Biol. 5: 784-790

Zhou L, Schnitzler A, Agapite J, Schwartz LM, Steller H and Nambu JR (1997) Cooperative functions of the reaper and head involution defective genes in the programmed cell death of Drosophila central nervous system midline cells. Proc Natl. Acad Sci. USA 94: 5131-5136 